\title{
A coping skills group and peer telephone support had similar effects on role performance, adaptability, and wellbeing in patients with multiple sclerosis
}

\author{
Schwartz CE. Teaching coping skills enhances quality of life more than peer support: results of a randomized trial with \\ multiple sclerosis patients. Health Psychology 1999 May;18:211-20.
}

QUESTION: For patients with multiple sclerosis (MS), is a coping skills group intervention more effective than telephone peer support for improving or maintaining long term psychosocial role performance, adaptability, and wellbeing?

\section{Design}

Randomised \{allocation not concealed\}*, unblinded, controlled trial with 2 years of follow up.

\section{Setting}

Boston, Massachusetts, USA

\section{Patients}

Patients were recruited from an MS clinic, newspapers, newsletters, and referrals. Inclusion criteria were neurologist confirmed diagnosis of MS and Expanded Disability Status Scale (EDSS) score 1-8.5 (minimal neurological problems to requirement of a wheelchair). 136 patients were randomised and 132 patients (mean age $43 \mathrm{y}, 74 \%$ women, mean duration of MS 8 y, $42 \%$ with progressive MS, mean income US $\$ 45000$ ) were assessed at 2 years.

\section{Intervention}

Randomisation was stratified by level of disability and course of disease. The coping skills group met for weekly 2 hour group sessions led by a health professional with a background in public health and clinical psychology. Patients attended 8 sessions and brought a support person of their choice for $\geq 3$ sessions on communication. Sessions were designed to teach methods of dealing with MS problems and goal setting. After 8 weeks, pairs of patients telephoned each other once a month for 10 months. The peer telephone support group employed lay people with MS who were trained to provide nondirective support (active listening but no offering of advice). 15 minute telephone calls were made monthly for 1 year. 64 patients in the coping skills group and 68 in the peer telephone support group completed the study.

\section{Main outcome measures}

Neurological disability using the EDSS and neuropsychological performance (3 tests) at 1 year to evaluate clinical health. Main outcomes were role performance (2 scales), adaptability (3 scales), and wellbeing (3 scales).

\section{Main results}

Analysis was by intention to treat. Both groups reported deterioration in neuropsychological functioning $(p<0.001)$ and a decreased sense of self efficacy in completing daily activities $(\mathrm{p}<0.05)$. Comparison of change over time between the 2 groups showed that patients in the coping skills group were less likely to cope by blaming others $(\mathrm{p}<0.01)$. The groups did not differ for any other outcomes.

\section{Conclusion}

A coping skills group intervention and peer telephone support had similar effects on role performance, adaptability, and wellbeing in patients with multiple sclerosis.

*Information provided by author.

\section{COMMENTARY}

Schwartz compared a face to face coping skills group with peer telephone support-2 services that are commonly provided by local chapters of MS societies. The study is unique in that it stratified for disability and disease course, examined many treatment effects over 2 years, and adjusted for neurological deterioration. This is essential for the appropriate evaluation of outcomes in a progressive disease such as MS. ${ }^{1}$

However, the author bases her conclusions on the differences within rather than between the 2 groups over time, which is not consistent with the study design. Although she found differences over time within both groups, only 1 significant difference was seen between groups: participants in the coping skills group used "blaming others" as a coping strategy less often than participants in the telephone support group.

The results are relevant to nurses working with individuals with MS in hospital and community settings. Specialisation of nursing in MS care exists internationally, and these results are particularly relevant to nurses specialising in this field. The results indicate that either support strategy will lead to similar results. However, nurses may want to consider compliance issues. 10 participants in the coping skills group dropped out primarily because of transportation issues, whereas only 2 participants in the peer telephone support group dropped out. The author noted that although the participants in the coping skills group were likely to miss only 1 of 8 group meetings, many received only half of the prescribed telephone calls, whereas many of the peer telephone support participants received the full dose of telephone calls.

Colleen Harris, RN, MN Clinical Practitioner Calgary Multiple Sclerosis Clinic Calgary, Alberta, Canada

Rudick R, Antel J, Confavreux C, et al. Clinical outcomes assessment in multiple sclerosis. Ann Neurol 1996;40:469-79.
Sources of funding: National Multiple Sclerosis Society; Fetzer Institute; Agency for Health Care Policy and Research; Franklin Institute.

For correspondence: Dr C E Schwartz, Frontier Science $\mathcal{E}^{\circ}$ Technology Research Foundation, Inc, 1244 Boylston Street, Suite 303, Chestnut Hill, $M A$ 02467, USA. Fax +1 5088561212. 